\title{
THE ELECTROENCEPHALOGRAM IN TRAUMATIC EPILEPSY
}

\author{
BY
}

\author{
Wing Commander, R.A.F.V.R., DENIS WILLIAMS
}

(From the Military Hospital (Head Injuries))

\author{
(RECEIVED 17TH OCTOBER, 1944)
}

THE material used in earlier studies of the immediate and remote effects of injury upon the human e.e.g. (Williams, 1941 (a) and (b)) gave little information upon the relationship of these effects to the development of traumatic epilepsy. In the three years which have since elapsed much more data have been accumulated on traumatic epilepsy, and more definite conclusions than were possible at that time can be made on this aspect of the subject. Other papers upon the general and focal changes in the e.e.g. associated with trauma have been published (Jasper, Kershman and Elvidge (1940) and Heppenstall and Hill (1943), and Jasper and Penfield (1943) have described changes associated with traumatic epilepsy. Reporting upon 81 patients studied by the e.e.g., of whom 32 had had a cerebral operation, they described focal abnormality in 90 per cent., with a generalized disturbance in the remainder. The most characteristic change in the e.e.g. in these cases was the presence of highvoltage spikes, although slower waves were also seen, and the authors commented upon the close association of the position of the observed cerebral lesion in the 32 operated cases, and the site of focal discharge in the e.e.g. They thought that the 10 per cent. of patients who did not show such a focus may have been suffering from essential, rather than traumatic, epilepsy. The importance of trauma in causing focal changes in the e.e.g. has also been established by Gibbs, Merritt and Gibbs (1943).

The present enquiry is not so much concerned with the focal nature of the changes in the e.e.g. in traumatic epilepsy as with the relationship of different abnormalities in the e.e.g. of patients with head injuries to the subsequent onset of traumatic epilepsy. Evidence based on the observation of a large number of subjects will be presented, and this evidence will be related to the possibility of predicting the onset of traumatic epilepsy by means of electroencephalographic examination.

\section{Method}

Material. There were in all 1,002 subjects who were divided upon clinical evidence into the following groups:

(1) 241 normal, healthy controls with no personal history of head injury, faints or fits;

(2) 234 patients with head injuries without epilepsy;

(3) 210 patients with traumatic epilepsy;

(4) 275 patients with apparently idiopathic epilepsy;

(5) 42 patients who were examined by the e.e.g. before and after the onset of traumatic epilepsy.
The subjects were all adults serving in the armed Forces, those with head injuries or epilepsy being hospital patients who were examined by electroencephalography during routine investigation. The normal controls were also service personnel of the same group, mainly derived from the staff of the hospital. An account of these normal subjects has already been given (Williams, 1944). The patients with head injuries were selected from several thousand examined during three years, but they were only specially selected in so far as those in whom there was doubt as to the presence of attacks, or as to the nature of the attacks, were rejected from the series. They were all patients in whom the severity of the injury or the persistence of symptoms had led to admission to a service hospital. They included patients with open and closed cranial injuries, and they were all investigated more than 4 weeks after the injury. The clinical and e.e.g. records of a sufficiently large number of consecutive cases were scrutinized to give adequate samples for statistical comparison, but the relative numbers do not reflect the actual incidence of epilepsy in the total series. These head injury patients, both with and without fits, were divided into those with closed and-penetrating injuries. The last group of patients, by their nature few in number, was followed up specially. These were patients who had had a head injury, had been fully investigated, and who had at a later date developed fits which led to their re-investigation. Particular importance was attached to the results in these patients.

All the groups were taken from the same restricted population of service men; the patients with traumatic epilepsy had been drawn from the same population of head injury patients as those without fits, and the last small group of traumatic epileptics only differed in that the patients had been examined before and after the onset of epilepsy. The groups may therefore be compared with each other with impunity.

Technique. The technique of recording, and the method of examination and of assessing the e.e.g. records, with a description of the different types of abnormality, has already been given in detail (Williams, 1941 (a) and 1944). In brief, records of at least 15 minutes duration were obtained from a three- or sixchannel Grass ink-writing electroencephalograph, two rows of saline pad electrodes being placed over each hemisphere. The electrodes were, as a routine, connected in pairs to give "bipolar" recording; some records were checked with simultaneous " monopolar" recording. The records were divided into normal and abnormal without knowledge of the clinical history of the patients, upon the basis of a norm which excludes 12 per cent. of the apparently normal group. The records classified abnormal were divided into three groups - those with characteristic epileptic outbursts (larval outbursts), those with other less well-defined paroxysmal disturbances, and those which although classified abnormal did not contain any episodic abnormal activity. Examples of these different types of abnormal e.e.gs. recorded in epileptic subjects have already been published (Williams, 1944, Williams and Sweet, 1944).

Having done this, features in the clinical story were related to the type of e.e.g. change which had been 
found. In most instances the subject was only investigated once, but in the group of patients in whom e.e.g. records had been obtained before the first fit, other records were obtained when possible at varying intervals of time after it.

\section{Results}

The distribution of normal and abnormal e.e.g's. in the five groups already described is shown in Table I. The percentages are corrected to the nearest whole figure.

TABLE I

\begin{tabular}{|c|c|c|c|c|c|}
\hline \multirow[b]{2}{*}{$\begin{array}{l}\text { Features in } \\
\text { the e.e.g. }\end{array}$} & \multicolumn{5}{|c|}{ Percentage of subjects } \\
\hline & $\begin{array}{c}1 \\
\begin{array}{c}\text { Nor- } \\
\text { mal }\end{array} \\
(241)\end{array}$ & $\begin{array}{c}2 \\
\text { Head } \\
\text { injury } \\
\text { with- } \\
\text { out } \\
\text { fits } \\
(234)\end{array}$ & $\begin{array}{c}3 \\
\\
\text { Head } \\
\text { injury } \\
\text { with } \\
\text { fits } \\
(210)\end{array}$ & $\begin{array}{c}4 \\
\text { Recorded } \\
\text { before } \\
\text { traumatic } \\
\text { epilepsy } \\
\text { began } \\
(42)\end{array}$ & $\begin{array}{c}5 \\
\text { Idio- } \\
\text { pathic } \\
\text { epilepsy } \\
(275)\end{array}$ \\
\hline $\begin{array}{l}\text { Larval epilep- } \\
\text { tic outbursts }\end{array}$ & 0 & 0 & $8 \cdot 6$ & 5 & 27 \\
\hline $\begin{array}{l}\text { Other par- } \\
\text { oxysmal out- } \\
\text { bursts } \quad .\end{array}$ & 0.8 & 14 & 17 & 18 & 29 \\
\hline $\begin{array}{l}\text { Other forms } \\
\text { of abnor- } \\
\text { mality .. }\end{array}$ & 11 & 46 & 37 & 38 & 19 \\
\hline $\begin{array}{c}\text { Total abnor- } \\
\text { mal } \\
\text { Normal }\end{array}$ & $\begin{array}{l}12 \\
88\end{array}$ & $\begin{array}{l}70 \\
40\end{array}$ & $\begin{array}{l}63 \\
37\end{array}$ & $\begin{array}{l}60 \\
40\end{array}$ & $\begin{array}{l}75 \\
25\end{array}$ \\
\hline
\end{tabular}

It will be seen in Table I that the proportion of subjects with different types of e.e.g. is about the same in the small group of head injury patients who were investigated before their first fit (column 4) as in those investigated after fits had commenced. (column 3). As the only difference between these two groups lies in the time of recording the e.e.g. they will for the moment be considered together as traumatic epileptics. The group of 42 patients with e.e.g's. before the first fit will be discussed in more detail later.

The distribution of the different forms of abnormality in the five groups of subjects is shown as a histogram in Fig. I. The following observations may be made upon the figures presented.

LARVAL EPILEPTIC OUTBURSTS are not found at all in two large groups of subjects who have not had epileptic fits. This is so whether or not a head injury has been sustained. On the other hand, 9 per cent. of traumatic epileptics and 27 per cent. of idiopathic epileptics show these outbursts. These differences are reliable statistically. It has been stated elsewhere (Williams, 1944) that larval epileptic outbursts are very rarely seen without overt fits, and that their presence indicates an established epileptic state. From columns 3 and 4 in Table $I$ it is clear that the 2 patients out of the 252 ( 5 per cent. of 42 in column 4) with a head injury who showed larval outbursts in the e.e.g. in the absence of fits, developed fits shortly afterwards. This indicates that the presence of these outbursts in a patient's e.e.g. record makes the

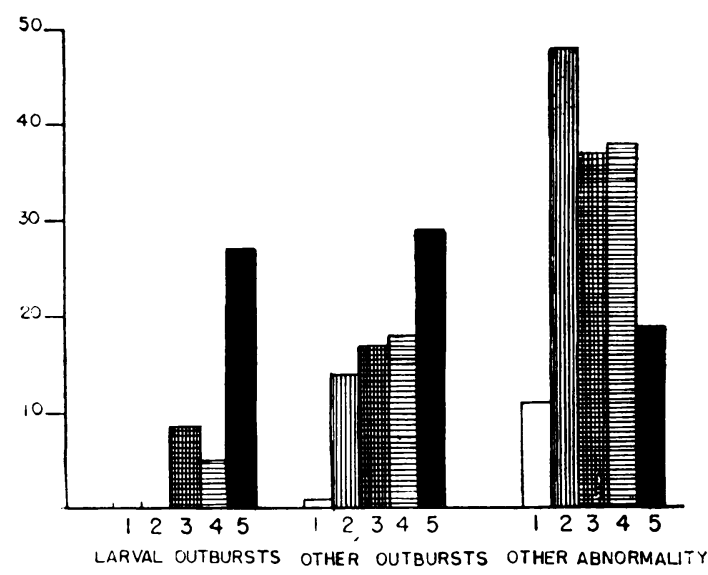

FIG. 1.-The figures 1 to 5 on the abscissa refer to the numbered columns in Table I, viz., 1 , normals; 2 , head injuries; 3 , traumatic epileptics; 4 , records made before the onset of traumatic epilepsy; 5, idiopathic epileptics; the ordinate represents the percentage of each group of cases.

likelihood of traumatic epilepsy very great indeed. Columns 3, 4 and 5 show that the chance of observing a larval epileptic outburst in the e.e.g. of idiopathic epileptics is three times as great as in traumatic epileptics.

OTHER PAROXYSMAL OUTBURSTS in the e.e.g. are distributed in the five columns in Table I in the same general manner as the larval outbursts, but the differences between the columns are not so distinct. Although these outbursts are uncommon in normal subjects, they are nearly as frequently seen in the group of head injury patients who have not developed fits as in those who have. It is certain, of course, that some of the first group will develop fits at a later date since the time of recording the e.e.g. after the injury was sometimes as short as a month, and was in most cases under a year. Nevertheless, it seems that the presence of paroxysmal outbursts in the post traumatic e.e.g. does not necessarily indicate the development of traumatic epilepsy, although it has been shown that in other pathological conditions these outbursts usually indicate an epileptic tendency (Williams, 1944). This statement is probably also true for head injuries, for it is likely that sometimes a liability to traumatic epilepsy which is reflected in the e.e.g., may not become overt in the form of fits for many years. In an earlier paper (Williams, 1941 (a)) it was shown that reversible changes in the e.e.g. recorded shortly after severe brain injury were indistinguishable from those seen in epileptics, and it seemed as though trauma gave rise to transient cerebral changes conducive to epilepsy which were reflected in the e.e.g. of many more subjects than developed traumatic epilepsy later. The clinical evidence of these changes is the early occurrence after a head injury of fits, which have no relationship to the later onset of traumatic epilepsy. It follows that in some traumatic epileptics other factors than the injury must be required to potentiate the latent epileptic liability. The development of these factors may 
account for the great delay in the onset of fits in some cases of head injury. This line of enquiry might well prove fruitful, but the results so far presented in Table I merely indicate that if these outbursts in the e.e.g., which are usually associated with epilepsy, occur more than a month after a head injury they are only associated with overt fits in a little more than half the cases. Conversely these changes occur in a sixth of the patients who develop traumatic epilepsy, compared with nearly a third of idiopathic epileptics.

Taking all the transient disturbances in the e.e.g. together it is seen that they occur in less than 1 per cent. of normals, in 14 per cent. of uncomplicated head injuries, 25 per cent. of traumatic epileptics and nearly 60 per cent. of idiopathic epileptics. Further information upon the relationship of these disturbances to the later onset of traumatic epilepsy can be obtained by determining the incidence of traumatic epilepsy in groups of patients with and without transient disturbances in the e.e.g. The results of such an enquiry in the present series of head injury cases are shown in Table II.

TABLE II

\begin{tabular}{|c|c|c|c|c|c|}
\hline \multirow{2}{*}{$\begin{array}{c}\text { Head } \\
\text { injury }\end{array}$} & \multirow{2}{*}{$\begin{array}{l}\text { E.E.G. } \\
\text { transient } \\
\text { outbursts }\end{array}$} & \multirow{2}{*}{$\begin{array}{c}\text { Number } \\
\text { of } \\
\text { cases }\end{array}$} & \multicolumn{2}{|c|}{$\begin{array}{l}\text { Traumatic } \\
\text { epileptics }\end{array}$} & \multirow{2}{*}{$x^{2}$} \\
\hline & & & Number & Per cent. & \\
\hline \multirow{2}{*}{ Closed } & 0 & 178 & 8 & $4 \cdot 5$ & \multirow{2}{*}{$8 \cdot 5$} \\
\hline & + & 35 & 7 & $20 \cdot 0$ & \\
\hline \multirow{2}{*}{ Open.. } & 0 & 75 & 6 & $8 \cdot 0$ & \multirow{2}{*}{$3 \cdot 8$} \\
\hline & + & 28 & 7 & $25 \cdot 0$ & \\
\hline
\end{tabular}

The probability that the differences in Table II are due to chance is less than 1 in 100 , and 1 in 20 respectively. The probability of both the differences between the groups with and without transient outbursts being due to chance is less than 1 in 100 . Table II therefore shows that there is greater risk of epilepsy after a head injury, whether closed or penetrating, if transient outbursts of abnormal waves are seen in the patient's e.e.g. The likelihood is increased in these cases by about 4 times.

Non-SPECIFIC ABNoRmality. The proportion of normal and epileptic subjects who are considered to have abnormal records has been discussed elsewhere (Williams, 1944) and has been investigated in detail by others, notably Jasper and Kersham (1941), Gibbs, Gibbs and Lennox (1943) and Finley and Dynes (1942). The total incidence of abnormality in the e.e.gs. of subjects with head injuries seems, from columns 2, 3 and 4 of Table I, to be unaffected by the presence of epilepsy. As the incidence of transient outbursts, and particularly of "larval" attacks, is greater in the epileptic group, it follows that abnormality of a non-specific kind alone is less commonly seen in them. The dysrhythmias of this indeterminate sort which are seen, are sometimes the result of the injury, and in others they represent evidence of pre-traumatic abnormality in the e.e.g. (Williams, 1941 (b); Heppenstall and Hill, 1943). Although groups of subjects with such abnormal e.e.gs. have been shown to contain a higher proportion of epileptics than is found in the population at large, the presence of this kind of abnormality in patients with head injury does not seem to be closely related to the likelihood of traumatic epilepsy. It must be borne in mind, however, that there is more likelihood of having both an abnormal e.e.g. and traumatic epilepsy after a severe head injury than after one of mild degree.

Closed v. Penetrating Injuries.-Table III shows that the incidence of paroxysmal outbursts of the kind usually associated with epilepsy are much more frequent in a group of patients with penetrating head injuries than in a similar group with closed head injuries. For this table $\chi^{2}$ is 18.4 and $\mathrm{P}$ is less than 0.01 , indicating that the likelihood of the difference being due to chance is less than 1 in 100 . The proportion with non-specific abnormality is by contrast about equal in the two groups. The incidence of traumatic epilepsy is higher when dural penetration has occurred (Ascroft, 1941), so that the relatively high incidence of " epileptic" changes in the e.e.g. in this series is explained. This high incidence without any change in other forms of abnormality might be expected from the results already presented.

TABLE III

\begin{tabular}{l|c|c|c|c|c}
\hline & & \multicolumn{3}{|c}{$\begin{array}{c}\text { E.E.G. findings } \\
\text { (percentage of total cases) }\end{array}$} \\
$\begin{array}{c}\text { Type of } \\
\text { injury }\end{array}$ & $\begin{array}{c}\text { Num- } \\
\text { ber of } \\
\text { cases }\end{array}$ & $\begin{array}{c}\text { Larval } \\
\text { out- } \\
\text { bursts }\end{array}$ & $\begin{array}{c}\text { Other } \\
\text { transient } \\
\text { out- } \\
\text { bursts }\end{array}$ & $\begin{array}{c}\text { Other } \\
\text { abnor- } \\
\text { malities }\end{array}$ & $\begin{array}{c}\text { Nor- } \\
\text { mal }\end{array}$ \\
\hline $\begin{array}{l}\text { Penetrating } \\
\text { Closed }\end{array}$ & 70 & 4 & 26 & 47 & 23 \\
\hline
\end{tabular}

To summarize the observations made so far: an abnormal e.e.g. persisting after a head injury does not necessarily increase the likelihood of traumatic epilepsy, but the presence of episodic outbursts of abnormal waves does. If these disturbances are organized into patterns considered to be larval attacks, epilepsy is almost certain to supervene, while transient disturbances of a less dramatic kind add materially to the likelihood of its onset.

E.E.Gs. Recorded beFore the ONSET OF TRAuMATIC EPILEPSY.--In all the 42 cases in column 4 of Table I the patient had been investigated while under treatment in hospital for the effects of a head injury. While still in hospital, or more commonly on return to duty, one or more epileptic fits had occurred and the patient was referred back to hospital. It was thus possible to investigate each of these cases before 
and after the onset of epileptic fits. The interval between the two investigations during which fits commenced ranged from 3 months to 3 years. The number of cases with different types of e.e.gs. recorded before the fits began is shown in column 4 of Table I, and, as already noted, is similar to the figures for e.e.gs. recorded after the onset of traumatic epilepsy

The changes observed between the records made before and after the fits began seemed to be unpredictable, but the numbers of cases with each kind of record were insufficiently large to have any comparative value alone. It was commonest for the e.e.g. to remain unchanged in any particular after the onset of traumatic epilepsy, whether it had previously been normal or abnormal, and whether or not it had contained episodic disturbances before the fits began. When changes occurred they were invariably caused by the appearance of episodic disturbances in a record which had previously been either normal or abnormal. Observation of these episodic disturbances in the e.e.g. record may have been to some extent governed by chance, and transient disturbances must have been missed because of the relatively brief period of recording which was dictated by the demands made upon the department. This would be particularly likely where the episodes were infrequent. Even so, disturbances of an epileptic type were seen in nearly a quarter of cases before fits began, and in some instances they preceded the first fit by more than a year.

Several samples of e.e.gs. recorded before and after the onset of symptomatic epilepsy have already been described at length (Williams, 1944). As the types of records seen in this series, and their relationship to the type of clinical fit, is the same as in those in the earlier report, further description is unnecessary.

FoCAL ABNormality.-A specially important point which has not been covered is the relationship of the position of origin of transient outbursts of an epileptic type to the focal abnormality seen in the e.e.g. of patients with penetrating wounds. Jasper and Penfield (1943) have stressed the frequent occurrence of foci of abnormal discharge in the e.e.g. of patients with traumatic epilepsy, and have shown the close relationship of the epileptic focus to a lesion which was demonstrated at operation in 30 out of 32 cases. Although the present investigation was not concerned primarily with the foci of discharge, such foci were found in a large proportion of cases of traumatic epilepsy, in contrast to the group of idiopathic epileptics. These findings coincide with those of Gibbs, Merritt and Gibbs (1943). Foci of non-specific activity are common after head injuries, and have been adequately described, Heppenstall and Hill (1943). When such abnormality existed in the present series in association with episodic "epileptic" activity, the sites of origin of the two kinds of discharge were often at or near the same point on the scalp. This focus was, however, in many cases merely a zone of maximal abnormal activity where fast waves ("spikes") could be picked up with greater facility than from elsewhere. Exploration of other points on the scalp showed that the discharge of spikes was widespread, with frequently another zone of increased abnormality on a symmetrical point on the other side of the scalp. Sometimes, however, the epileptic discharge would not coincide with the area of gross brain damage. For instant, bifrontal discharge might accompany an area of slow activity elsewhere (Case 2).

The relationship which the slow abnormal waves of cerebral damage bears to the episodes of faster, high voltage waves of epilepsy should be considered in time as well as in space. It might be expected that they would relate spatially, but a close temporal relationship cannot be found, while the possibility of abnormality of the episodic type having been present in the pre-traumatic e.e.g. further confuses the picture.

Immediately after a head injury it is usual to find gross abnormality characteristic of severe cerebral damage which during the period of resolution may mimic the picture of epilepsy, but which in a few weeks subsides with the gradual reappearance of normal rhythms (Williams, 1941 (a)). For this reason all the cases of head injury included in this series were examined more than four weeks after injury. At some point in this period of recovery the other kind of abnormality appears, but the time of its appearance is very variable and its relationship in time to the onset of traumatic epilepsy is also variable. Table I shows that episodic activity is less than half as common in traumatic epilepsy than in idiopathic, and it is certainly very difficult to observe any abnormality in the e.e.g. of some traumatic epileptics in spite of frequent recording between seizures. Therefore, it is likely that, in some instances, the first demonstrable sign of the development of the epileptic state will be an overt fit. Episodic changes appearing in the e.e.g. before the first fit are nevertheless common, and by serial recording over months the process has been closely observed. In these cases it is evident that months after the injury an abnormal state is established which steadily increases until it finds expression first in an indefinite dysrhythmia, later in short episodes of abnormality, later in larval epileptic outbursts of the more clearly defined sort, and finally in a frank epileptic fit.

This process is most easily seen in penetrating wounds, for the disturbance can be studied in the neighbourhood of the local injury. Case 1 is an excellent example of it.

Case 1.-A gunner, aged 21, sustained a penetrating wound of the right frontal lobe as a result of a bomb explosion on 9th April, 1941. He remembered the whistle of the bomb before he was struck, but had a retrograde and post traumatic amnesia of about a second. He remained perfectly alert afterwards.

Nine hours later he was fully examined in hospital. There was a large, jagged wound of the right frontal region with shattered bone and much brain tissue extruding through it. Full neurological examination failed to 
show any disturbance of function. He was well-orientated and fully conscious. To special testing there was slight defect of recall and of judgment. X-ray showed that a large depressed fracture of the right frontal bone with many fragments driven into the right frontal lobe. A piece of bomb casing was lying just above the left supraorbital ridge, having probably traversed both the right and left frontal obes.

An e.e.g. recorded 10 hours after the injury was grossly abnormal. The report follows: "The e.e.g. was grossly abnormal. Electrodes were placed 2 inches in front of and 2 inches behind the scalp wound and in corresponding positions on the left side. From these positions rhythmic bursts of high voltage pure sine waves with a frequency of 2 a second were obtained. These outbursts were indistinguishable from subclinical epileptic outbursts that have been found in other cases of severe cerebral damage when a record is obtained within a few hours of the injury. When they occurred these outbursts were symmetrically distributed in the frontal poles but between them the record was more abnormal on the right side than on the left. Comparing the anterior and posterior electrodes it was evident that this abnormality was present in the frontal poles but not behind the wound. A sterile stainless steel electrode placed in the wound showed a focus of discharge from the area under the bone defect." Samples of the records are shown in Figs. 2 and 3. Toilet of the wound was carried out by Lt.-Col. E. H. Botterell, R.C.A.M.C. The scalp wound was excised, the bone defect enlarged, and all loose bone fragments removed. Ventricular fluid welled into the brain defect which was $2 \frac{1}{2}$ inches deep. After a clean field surrounded by apparently healthy brain was obtained the wound was dusted with sulphanilamide and completely closed. The projectile was left in situ.

Progress. The patient was a little drowsy but was fairly well-orientated during the next three days, and apart from a bifrontal headache he had no complaints. There were at no time any signs of disturbance of motor or sensory function. Daily lumbar puncture produced blood-stained fluid under a pressure of $300 \mathrm{~mm}$. of cerebro-spinal fluid.

An e.e.g. on the third day showed no substantial change.

Four days after the injury a mild right facial palsy of upper neurone type was noticed and the patient was restless. He had been incontinent of urine three times.

A week later the wound had healed by first intention, the cerebro-spinal fluid was straw coloured, and there were no neurological signs. Psychometric testing showed that he was alert, fairly well-orientated and had normal powers of recall. His reasoning, judgment and arithmetical ability were slightly defective and he was thought to be too apathetic and passive.

Two weeks after the injury he was up and about the ward, symptom and sign free, but still showing evidence of defect of reasoning, calculation and judgment. $\mathrm{He}$ had been incontinent nine times since the injury, saying characteristically that he "could not be bothered to ask for a bottle." He was quite contented to be in a wet bed.

An e.e.g. 14 days after injury was reported upon as follows: "Records taken from both frontal lobes still show very considerable abnormality consisting of highvoltage 1 to 3 a second waves. This is much worse on the right side than on the left and a very beautiful phase reversal occurs when an electrode is placed a little to the right of the midline. This indicates that a focus of discharge of abnormal electrical activity is starting in this region. The episodes of high-voltage sine waves resembling epileptic outbursts have now disappeared "'(Fig. 3).

Three weeks after the injury an air-encephalogram showed that the body of the right lateral ventricle was being pulled out toward the bone defect.

Five weeks after injury surgical repair of the bone defect was successfully undertaken. The dura was found to have regenerated considerably, and the area of exposed brain was only the size of a shilling.

Nine weeks after the injury detailed psychiatric testing failed to show any abnormality.

An e.e.e. recorded at this time was "well within normal limits throughout the hemispheres except for a small area in the right frontal lobe below the wound of entry. The abnormality in this area is slight and very well circumscribed" (Fig. 2 and 3 ).

Fourteen weeks after the injury he returned to duty. Twenty- two weeks after injury he had a tonic-clonic fit with tongue biting and incontinence. He had one more in the next year, and then 4 while asleep in the same day in rapid succession. All of the attacks were witnessed and more were focal.

Two years after injury he was re-examined in hospital

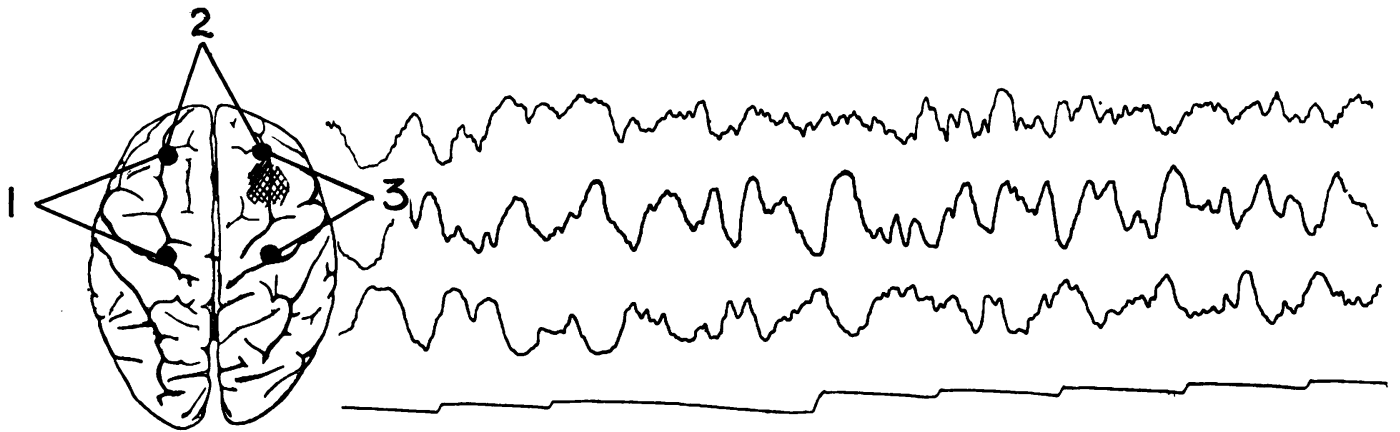

TEN HOURS AFTER INJURY

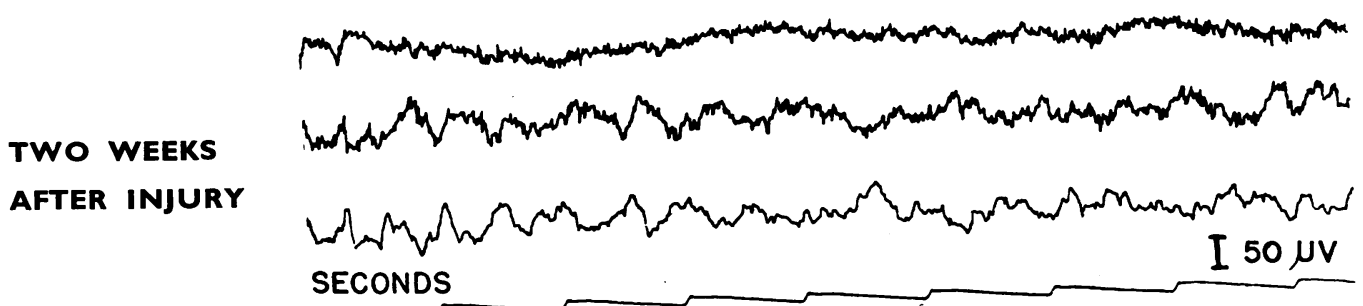

FIG. 2.-e.e.g. records from Case 1. The electrode placements are shown in the diagram, where the shaded area represents the position of surface injury to the brain. Contemporary descriptions of the records from which the samples in this and other figures have been taken appear in the case reports. The figure shows the subsidence with time of high voltage slow waves, the phase reversal in the lower two tracings indicating that the waves are emanating from the area of brain damage. 


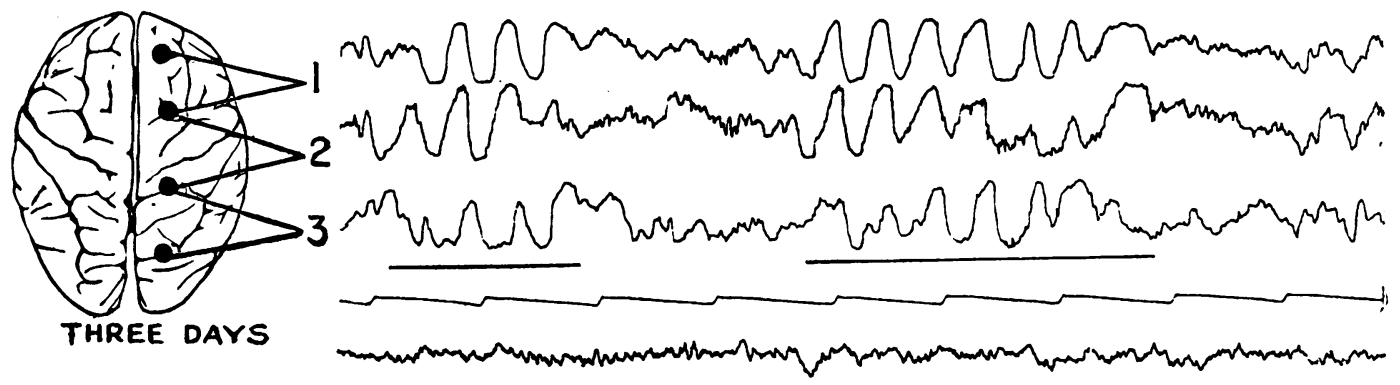

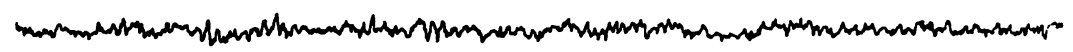

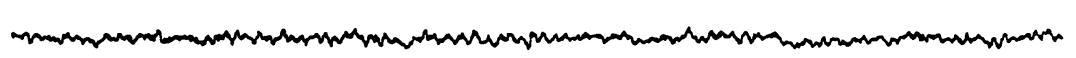

TWO MONTHS

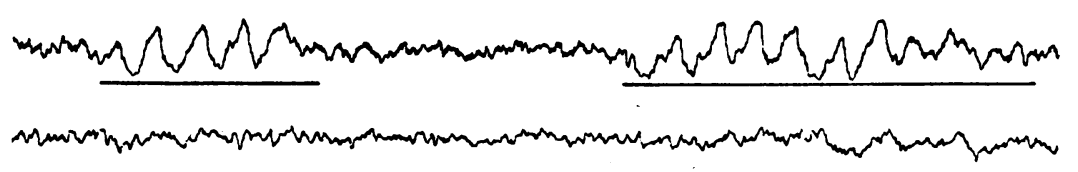

TWO YEARS

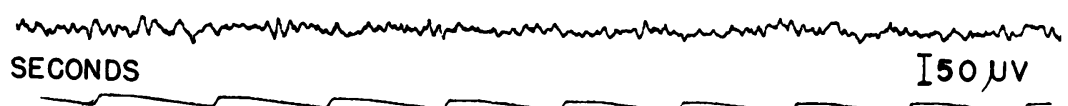

Fig. 3.-Records from Case 1 with routine electrode placements (cf. Fig. 2), 3 days, 2 months, and 2 years after injury, chosen to show the initial improvement and subsequent deterioration in the e.e.g. High-voltage slow waves, indistinguishable from those of epilepsy, are evident in all leads 3 days after the injury. These disappear with clinical recovery, and after two months the physiological dominant frequency is beginning to return. At this time low voltage 3 a second waves are limited to the frontal lobe. After 2 years bursts of high voltage 3 a second waves, of an epileptic kind, are obtained from the neighbourhood of the injury.

and there were no abnormal signs in the central nervous system. Psychiatric examination failed to show any defect in intellect or deterioration in personality. The cerebro-spinal fluid was normal in all respects, and the ventricular outline had not changed appreciably since the previous encephalogram taken 2 years before. The personal and family history revealed nothing of notethere was no evidence of epilepsy or migraine.

An e.e.g. at this time (11.3.43) showed " outbursts of high-voltage rhythmic slow waves of 2 to 4 a second which were considerably increased by overbreathing, but which were confined to the right frontal lobe especially under the bone defect. Records from positions elsewhere on the scalp were considered normal " (Fig. 3). The outbursts were thought to be epileptic in type.

COMMENT.-This is an example of severe laceration of the frontal lobes which healed by first intention after operation. Grand mal fits began 5 months after injury. The e.e.g. immediately after injury showed severe focal abnormality with generalized outbursts of waves indistinguishable from those seen in epilepsy. This abnormality subsided and except for slight focal abnormality of a non-specific kind the e.e.g. returned to normal. Subsequent records showed the development of typically epileptic discharges in the region of the original injury.

The focal epileptic disturbance, such as that seen in Case 1, may become widespread throughout the hemispheres, and when this has happened, even if a focus of discharge is evident, removal of that focus and of the damaged brain does not necessarily interrupt the epileptic process. This fact is shown in Case 2.
Case 2.-A signalman, aged 34 , was struck in the right posterior parietal region by a piece of mortar bomb on 20th November, 1942. This entered the skull and passed through the brain to come to rest against the falx just anterior to the corpus callosum. It was subsequently shown that the site of entry was in the region of the angular gyrus on the right side. There was no amnesia and the patient walked back for treatment. He complained of diplopia and slight impairment of vision at that time. Rough examination shortly after the injury showed reduction of the left half of the visual fields without any other abnormal signs in the central nervous system.

Two weeks after injury he had slight bilateral papillœdema with hæmorrhage.

Four weeks after injury he reached an evacuation hospital where depressed fragments of bone were elevated and superficial necrotic brain was removed under local anæsthesia. Neither the foreign body nor the indriven bone fragments were touched.

Eight weeks after injury bilateral papillœdema was still present with hæmorrhage in the left retina. External ocular movements were full and except for some enlargement of the blindspots the visual fields were full.

Sixteen weeks after injury he was admitted to the Military Hospital for Head Injuries. He had a simple defect of accommodation-convergence but no other abnormal signs were found on full examination of the nervous system. Intellectually he was normal, and there was no evident personality disorder. In particular the optic discs and visual fields were normal. Lumbar puncture showed no abnormality. X-ray showed a circular bone defect in the right posterior parietal region, $1.5 \mathrm{~cm}$. in diameter, with two small fragments of bone deep into it and a small metallic body in the midline just behind the sella.

An e.e.g. 4 months after injury (12.3.43). The report reads: "Bursts of abnormally fast waves with a frequency of 20 cycles were seen in the anterior parts of the hemispheres. They were more evident on the right 

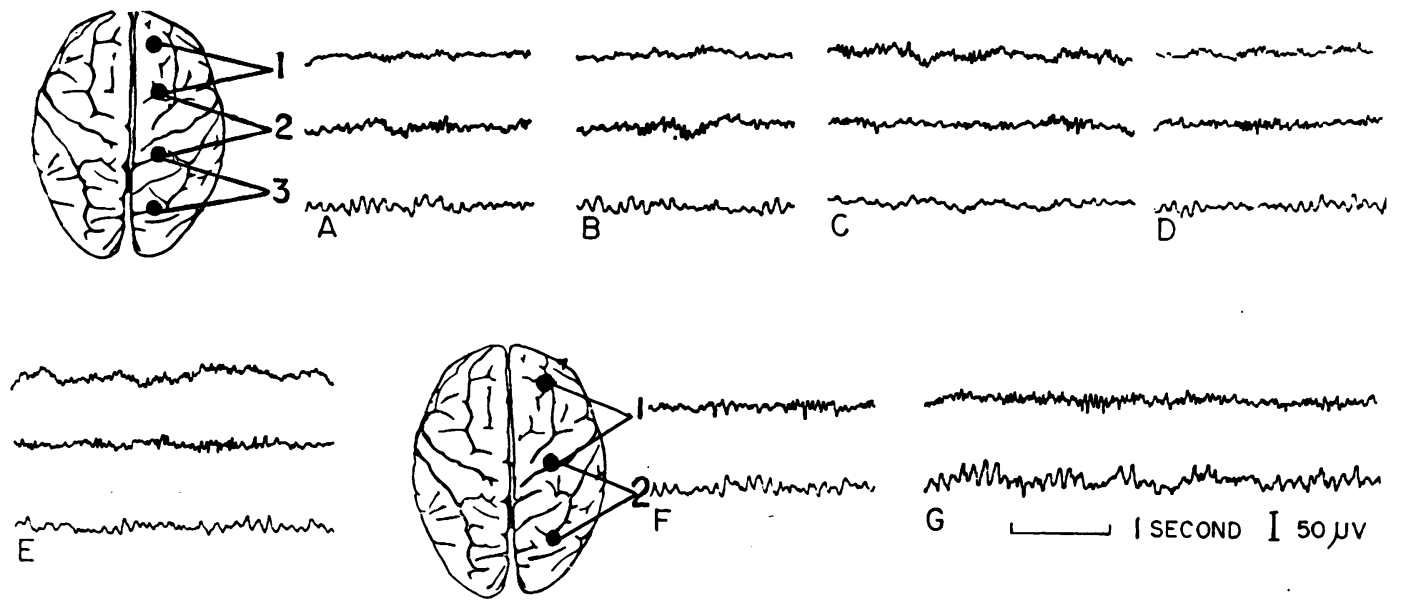

FIG. 4.-Samples of records obtained at different times from Case 2. The times of recording were as follows : A, 4 months after injury and 4 months before the first fit; B, 8 months after injury and 2 days after the first fit ; $\mathrm{C}$ and $\mathrm{D}$, immediately before and 10 days after removal of the cerebral cicatrix; E, F, and G, 4, 5, and 6 weeks after the operation. All samples show outbursts of fairly high voltage abnormally fast waves, lasting for 1 to 2 seconds and confined to the anterior part of the hemisphere. They were thought to represent epileptic outbursts, and were symmetrically distributed. They were not affected by removal of the scarred brain. Paper speed and amplification are constant.

side, where their maximum voltage was 30 microvolts. There were persistent abnormal waves with a sine form and with frequencies varying from 0.5 to 4 a second underlying a stable dominant frequency of 10 cycles in the neighbourhood of the scalp wound. These represented local cerebral damage of moderate severity. The frontal outburst of fast waves represented epileptic activity" (Fig. 4a). With orthoptic exercises the convergence-accommodation defect cleared up. As the wound was well healed and the patient was symptom and sign free it was decided not to explore the bone defect or to remove the indriven fragments. As he had had no fits he was discharged to duty.

Seven months after injury (6.7.43) he had a generalized tonic-clonic fit, witnessed by his wife. In it the head and eyes were turned to the side of the injury, but there were no other localizing features to be seen. He was readmitted to hospital (8.7.43).

An e.e.g. was recorded 2 days after the fit (8.7.43). The report was as follows: "The generalized episodic fast activity, thought to be epileptic in nature four months ago, is still present and is more evident. The focal disturbance consisting of slow sine waves has also persisted and is unchanged" (Fig. 4b).

Another series of records were made just before operation (23.7.43). The report read: " Careful exploration of the right hemisphere before operation showed that the abnormal slow waves of cerebral damage were strictly confined to the region of the bone defect. Phase reversals could be obtained for all these abnormal waves when an electrode was placed over the defect. The edge of the area of abnormal discharge was sharply defined, and the abnormal waves were being propagated radially from the site of the injury. There was no relationship between the site of injury and the position of the maximal epileptic discharges except that these discharges had a higher voltage on the affected side" (Fig. 4c).

Three weeks after the fit (26.7.43) the scar and indriven bone fragments were removed. At operation (Major C. A. Calvert) a right lateral bone flap was turned with the penetrating wound in its centre. The dura, which was adherent to the brain around the wound, was reflected and a large central scar was found. The arachnoid was very vascular in this region and there were dense vascular adhesions between arachnoid and cortex over the scar while a large cortical artery was firmly adherent to it. Beneath this there was a circular yellow gelatinous cortical scar $2.5 \mathrm{~cm}$. in diameter. This scar was depressed almost $0.5 \mathrm{~cm}$. below the surface 0 the surrounding normal brain. It was quite inactive to stimulation with a thyratron stimulator. The surrounding cortex, thought to be in the region of the supra marginal and angular gyri, was also inactive. The cortex was incised around the scar which was then completely excised. The scarred brain was cone shaped, the base of the cone extending to the trigone of the right lateral ventricle which was opened during the excision. Cerebro-spinal fluid welled freely into the wound and the opening was seen to be about $0.7 \mathrm{~cm}$. in diameter. A clean, dry field was obtained and the wound closed in the ordinary way. There was no disturbance of sensory, motor or visual functions immediately after operation, and recovery was uninterrupted.

An e.e.g. which was recorded 10 days after operation (6.8.43) showed : "The episodes of epileptic fast waves in the hemispheres, described earlier, were unchanged. Low-voltage 1 and 2 cycle sine waves were obtained from a small area in the middle of the skin flap. No phase reversal was found " (Fig. 4d). Records were repeated at weekly intervals until his discharge on September 15th, 10 months after the injury and 2 months after the fit. In all 10 sets of records were obtained. No further fits had occurred up to a year after the operation.

COMMENT.-This is a case of a penetrating wound of the brain without any evidence of general commotio cerebri. There was only transient disturbance of visual function. One e.e.g. recorded 4 months after the injury, when the patient was symptom and sign free, showed bilateral generalized fast activity of an epileptic type with abnormal slow waves in the neighbourhood of the injury. There was no focal epileptic disturbance around this area. Traumatic epilepsy began about 8 months after the injury and a large cortical scar was completely removed shortly after this. Serial e.e.gs. taken at frequent intervals before and after operation failed to show any subsidence in the epileptic activity which had been present before the fit. The epileptic process which had become generalized was not modified by removal of the causal abnormal brain.

Observation of the progress of this kind of 
case suggests that, as occurred in Case 2, when e.e.g. evidence of epileptic activity transcends the neighbourhood of local cerebral damage and becomes widepread it is likely to persist and not to be permanently affected by removal of the damaged and scarred brain which in some way initiates the epileptic processes. It seems, however, that when the damaged brain is completely removed before the process begins, and the e.e.g. recorded from the affected neighbourhood is normal, subsequent dysrhythmia is unlikely to appear. The evidence for this statement is obtained from many cases of penetrating brain injury in which toilet and debridement has been adequate. These cases, operated on when in the Military Hospital (Head Injuries) and 1st Canadian Neurological Hospital, have been investigated at varying intervals after the toilet, and it is very common to find no evidence of focal abnormality in the e.e.g. It seems that simple absence of brain tissue with slight gliosis is insufficent to give rise either to demonstrable slow activity or to a disturbance in the e.e.g. of the epileptic type. These two observations seem important in relation to the surgical management of traumatic epilepsy.

\section{Discussion}

Walter et al. (1937) pointed out that the e.e.g. was more likely to show abnormality in idiopathic epileptics than in those with symptomatic epilepsy, and they were unable to find any dysrhythmia in 4 cases of traumatic epilepsy. They also observed that the occurrence of abnormality in the e.e.g. of epileptics decreased with age. Jasper, Kershman and Elvidge (1940) and Gibbs, Merritt and Gibbs (1943), using a monopolar technique, found foci of abnormality so frequently in patients with traumatic epilepsy, contrasted with idiopathic epilepsy, that it seemed reasonable to conclude that a focal disturbance indicated a gross anatomical cause for the epilepsy. Paradoxically, although the e.e.g. can be used in this way to demonstrate unsuspected gross causes of epilepsy, it also indicates a fundamental unity between idiopathic and symptomatic epilepsy, and exposes the artificial nature of this division, for the pattern of the dysrhythmias seen are identical in the two conditions.

The immediate disturbances in the e.e.g. which result directly from cerebral injury caused by trauma, a tumour or any other agent, and those which arise subsequently and which are so frequently associated with epilepsy, can often be clearly differentiated. The directly acquired disturbances which were originally described by Berger (1929) and which were called " delta" waves by Walter (1937), have a frequency of the order of $\frac{1}{2}$ to 3 a second and are found in the neighbourhood of the damaged brain. When, however, all the hemisphere is in a state of dysfunction, as it is after concussion, the disturbance is widespread (Williams and Denny-Brown, 1941). The dysrhythmia caused by these slow waves is reversible, for it subsides with recovery, and the voltage, period, persistance and area of origin of the abnormal waves are directly related to the degree of cerebral dysfunction.

The secondarily acquired dysrhythmias which arise as an indirect result of the cerebral insult and which are the subject of this paper, have many characteristics by which they can be recognized. Their most fundamental quality is that their relationship to the injury is indefinite. Their morphological characteristics-voltage, period, distribution and number-do not reflect the severity of the injury; they may arise long after the immedate effects of injury have subsided; they may arise from points in the hemispheres remote from the damaged area; and their actual presence does not depend upon the severity or kind of injury alone. Another fundamental distinction between the primary and secondary effects which may arise in the e.e.g. as a result of cerebral damage is that of persistence in time. Whereas the direct effects are usually reversible and transient, subsiding with clinical recovery, the delayed indirect effects when they arise are usually irreversible and persistent.

The form of the indirectly acquired dysrhythmias depends, of course, upon the characters of the waves of which they are constituted. These waves tend to have a high voltage in relation to their period; their frequency ranges from 2 to 3 a second upwards; there is great variability both in frequency and amplitude so that the record gives an appearance of instability; they tend to be episodic in their occurrence and to be linked into wave complexes of easily recognized form; they are often symmetrical or widespread throughout the hemispheres although they may be propagated from the zone of damaged brain over long periods of time; and they respond to physiological changes such as visual stimulation or changes in the blood $\mathrm{Ph}$. It is evident then that a distinction is being made, and can usually be made as a result of a single examination of the e.e.g. records, between the primary effects of brain damage, which are reversible and transient, and secondary effects which are irreversible and persistent. This distinction has been made in presenting the results of the present investigation.

It will be recognized that the secondary changes may be indistinguishable from the disturbances seen in the e.e.g. of patients with idiopathic epilepsy and with other behaviour disorders which appear to arise mainly from inborn, constitutional causes. The initiation of this kind of disturbance after brain damage certainly does not depend upon the damage alone. Other factors, some of which precede the trauma, are responsible. That the age of the patient is one of these factors is shown by an interesting discrepancy between the present observations and those of Gibbs, Merritt and Gibbs (1943). Whereas Gibbs and his colleagues show several illustrations of "wave and spike" complexes which occurred in children with traumatic epilepsy, the present writer had formed the opinion that this phenomenon was not encountered in symptomatic epilepsy The explanation seems to rest in the age groups, since none of the subjects of this present investigation are 
under 18 years, and children are rarely examined. Further evidence that other potent causes are at work is found in the observation that there is no obvious relationship between the nature or the site of brain damage-whether the injury is caused by trauma, tumour, infection or toxæmia-and the kind of epileptic disturbance which may result.

There is much histological evidence that the widespread pathological changes which occur in association with idiopathic and symptomatic epilepsy are similar, but, as Muskens (1928) concludes in his careful appraisal of the evidence, it is impossible to decide whether these well-recognized areas of neuronal degeneration and glial overgrowth reflect the result or the cause of long-standing epilepsy. The same is unfortunately true of the dysrhythmias seen in the e.e.g. It seems, in fact, that the purely descriptive approach to the electrical changes in epilepsy has reached the same point as the histological, a point which is far short of an understanding of the underlying disturbance which constitutes epilepsy, and further advances will depend on experimental methods.

Although the electrical disturbances associated with epileptic activity are similar whatever may be the precipitating cause of the illness, idiopathic and traumatic epilepsy differ in the frequency of the occurrence of the episodic discharges as well as in the site of origin of the discharges. These differences have some value in diagnosis.

When changes which have been described as larval attacks are seen at an interval after injury, epilepsy is virtually certain to supervene. When less distinct paroxysmal discharges occur there is more than an even chance of traumatic epilepsy, but in all other cases the e.e.g. gives no guide as to the probability of epilepsy following cerebral injury.

\section{Summary}

Electroencephalograms have been recorded in large groups of patients with idiopathic epilepsy, traumatic epilepsy and with head injuries without epilepsy, as well as in a large control group of normal subjects. e.e.gs. were also obtained before and after the onset of traumatic epilepsy in a small group of subjects.

The records in these groups have been compared, and the results obtained show that larval epileptic outbursts in the e.e.g. are invariably associated with overt fits, while less well-defined paroxysmal outbursts are also frequently associated with fits. These disturbances, which occur in 25 per cent. of traumatic epileptics compared with 56 per cent. of idiopathic epileptics, may be used with other signs to predict the likelihood of traumatic epilepsy. In other cases of head injury when these changes are absent, the e.e.g. offers no such help. The likelihood of epilepsy supervening when these disturbances are present has been examined in closed and penetrating head injuries.

The records made before and after the onset of fits show that the characteristic episodic changes often appear in the e.e.g. before the onset of traumatic epilepsy.

The site and nature of the disturbances in traumatic epilepsy are described. A distinction is made between the immediate and direct effects of trauma upon the e.e.g., and the remote, indirect and persistent effects which are usually associated with epilepsy. The results are compared with those of other workers and are used in discussing the relationship of symptomatic to idiopathic epilepsy.

I am grateful to the Medical Officers whose patients have been used for this enquiry, and to $A / S / O ~ J . K$. Reynell for her technical help. The apparatus used for many of the earlier cases was kindly lent to the Royal Air Force by the National Hospital, Queen Square.

\section{BiBliogRAPHY}

Ascroft, P. B. (1941). Brit. med. J., 1, 739.

Berger, H. (1929). Arch. f. Psychiat., 87, 527.

Finley, K. H., and Dynes, J. B. (1942). Brain, 65, 256.

Gibbs, F. A., Gibbs, E. L., and Lennox, W. G. (1943). Arch. Neurol. Psychiat. (Chicago), 50, 111.

Gibbs, E. L., Merritt, H. H., and Gibbs, F. A. (1943). Ibid., 49, 793.

Golla, F., Graham, S., and Walter, W. G. (1937). J. ment. Sc., 83, 137.

Heppenstall, M., E. and Hill, D. (1943). Lancet, i, 261.

Jasper, H. H., Kershman, J., and Elvidge, A. (1940). Arch. Neurol. Psychiat. (Chicago), 44, 328. and Kershman, J. (1941). Ibid., 45, 903.

and Penfield, W. (1943). Am. J. Psychiat., 100, 365.

Muskens, L. J. J. (1928). Epilepsy-Comparative pathogenesis, Symptoms and Treatment. London, Chapter 13.

Walter, W. G. (1937). Proc. roy. Soc. Med., 30, 579.

Williams, D. (1941 (a)). J. Neurol. Psychiat., 4, 107. (1941 (b)). Ibid., 4, 131.

and Denny-Brown, D. (1941). Brain, 64, 223.

(1944). Ibid., 67, 10.

and Sweet, W. (1944). Lancet, ii, 430. 